\title{
Robotic transthoracic diaphragmatic hernia repair
}

\author{
Antonios C. Sideris, MD, and Daniela Molena, MD, New York, NY
}

\footnotetext{
From the Thoracic Service, Department of Surgery, Memorial Sloan Kettering Cancer Center, New York, NY. This work was supported by the National Institutes of Health/National Cancer Institute (P30 CA008748).

Disclosures: D.M. is a consultant for Johnson \& Johnson, Urogen, Intuitive, and Boston Scientific. A.C.S. reported no conflicts of interest.

The Journal policy requires editors and reviewers to disclose conflicts of interest and to decline handling or reviewing manuscripts for which they may have a conflict of interest. The editors and reviewers of this article have no conflicts of interest.

Received for publication March 1, 2021; accepted for publication Aug 16, 2021; available ahead of print Aug 21, 2021.

Address for reprints: Daniela Molena, MD, Thoracic Service, Department of Surgery, Memorial Sloan Kettering Cancer Center, 1275 York Ave, New York, NY 10065 (E-mail: molenad@mskcc.org).

JTCVS Techniques 2021;10:578-80

2666-2507

Copyright (C) 2021 The Author(s). Published by Elsevier Inc. on behalf of The American Association for Thoracic Surgery. This is an open access article under the CC BY-NC-ND license (http://creativecommons.org/licenses/bync-nd/4.0/).

https://doi.org/10.1016/j.xjtc.2021.08.026
}

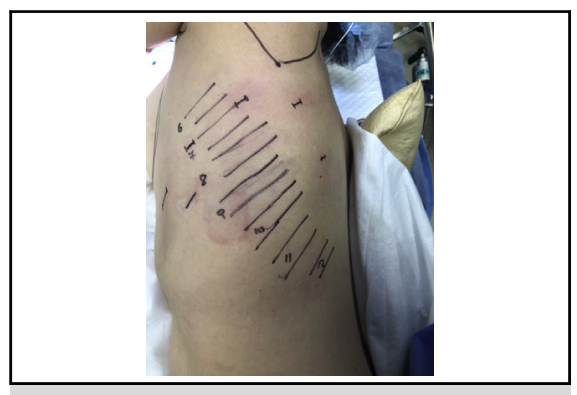

Port position for robotic diaphragmatic hernia repair.

CENTRAL MESSAGE

Robotic transthoracic repair is a useful approach for diaphragmatic hernia repair in the presence of a hostile abdomen.

See Commentaries on pages 581 and 582 .

Video clip is available online.

Diaphragmatic hernias are rare entities that can be congenital, traumatic, or iatrogenic. ${ }^{1}$ Transthoracic hernia repairs are most often performed via open thoracotomy, with few reports of robotic-assisted approaches. ${ }^{1-3}$ We report a case that illustrates our technique of transthoracic diaphragmatic hernia repair with mesh using the da Vinci $\mathrm{Xi}$ robotic platform in the presence of an extremely hostile abdomen. The Memorial Sloan Kettering Cancer Center institutional review board approved the submission, as this does not constitute human subjects research and the project does not involve identifiable patient information (\#16-1631, October 27, 2020). The patient gave informed written consent for the publication of the study data.

\section{TECHNIQUE}

A 41-year-old woman with stage IV high-grade serous ovarian carcinoma was referred to our clinic. The disease involved bilateral diaphragms, the posterior aspect of the liver, the inferior vena cava, and Morison's pouch. Additional findings were diffuse peritoneal carcinomatosis and large bilateral ovarian masses, disease along the bladder and uterosacral ligaments, and cancerous nodules on the surface of a 4-cm segment of colon. She underwent radical total abdominal hysterectomy with bilateral salpingooophorectomy, omentectomy, bilateral diaphragmatic stripping, appendectomy, and total peritoneal stripping. On 6-month computed tomography of the chest, abdomen, and pelvis, a new giant left diaphragmatic hernia was noted with distended stomach in the left chest, for which she was referred to thoracic surgery for repair (Figure 1).

We used the da Vinci Xi robotic system (Intuitive Surgical, Sunnyvale, Calif) with a 0-degree camera to minimize pressure on the intercostal nerve. Before incision, an esophagogastroscopy was performed, and the stomach was decompressed. Placement of an orogastric tube ensured continued gastric decompression and facilitated visualization during the operation. Port placement is shown in Figure 2. The camera was introduced in the sixth intercostal space at the posterior axillary line. Targeting of the platform was performed to the hernia defect. The AirSeal system (CONMED, Utica, NY) was introduced at the eighth intercostal space as anterior as possible for smoke evacuation and insufflation. A monopolar spatula was inserted through an $8-\mathrm{mm}$ port at the sixth interspace at the anterior axillary line, and fenestrated bipolar forceps with bipolar cautery were placed through an 8-mm port at the seventh intercostal space posterior to the tip of the scapula. We used 3 arms and an assistant port for our dissection; however, the addition of a fourth retracting arm posteriorly near the diaphragm can facilitate dissection and exposure.

The patient was placed in reverse Trendelenburg position at 30 degrees to facilitate reduction of abdominal contents. The abdominal viscera were mobilized and reduced into 

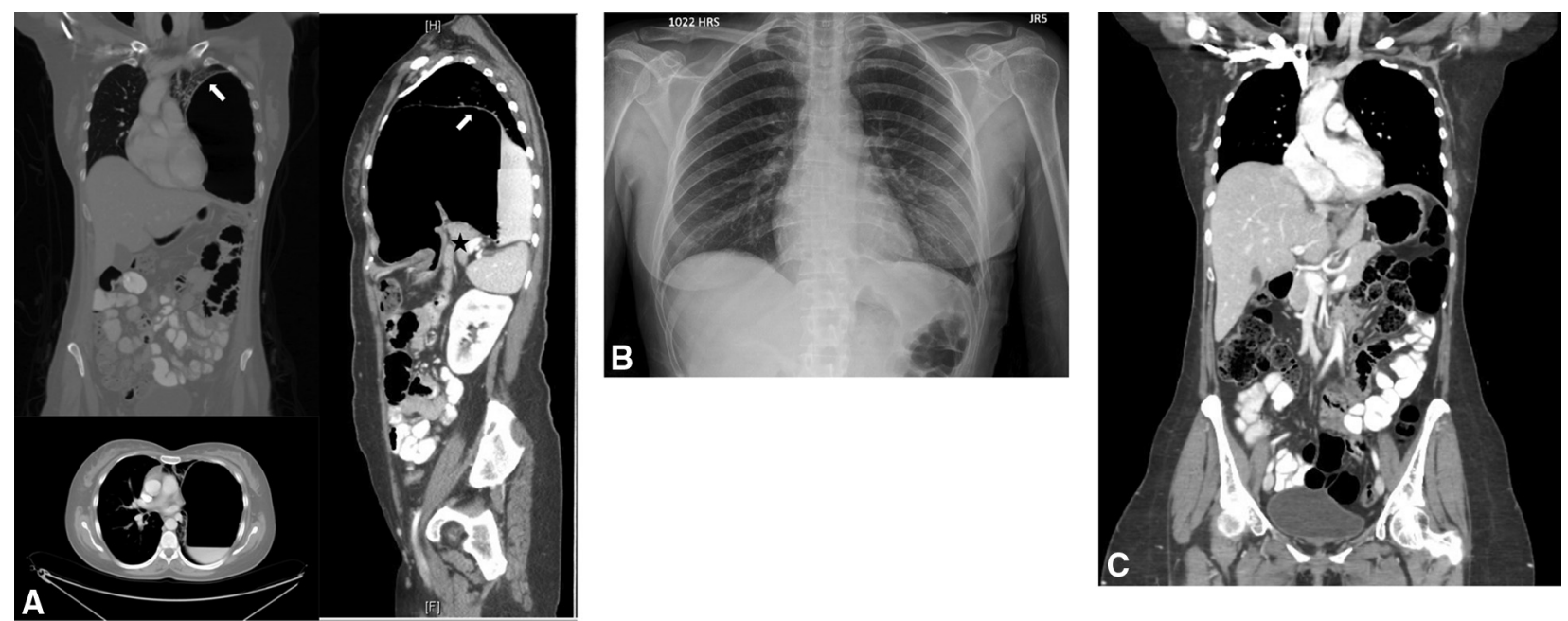

FIGURE 1. Giant left diaphragmatic hernia containing the majority of the stomach, with additional portions of the colon (the star depicts hernia, and white arrows depict the edge of stomach). A, Postoperative imaging showed no recurrence of the diaphragmatic hernia at 1 month (B) and 6 months (C) after the repair, without evidence of recurrence.

the abdomen. Positioning and insufflation can facilitate reduction, which can be challenging if there is loss of domain from a long-standing hernia. A 7-cm $\times 5$-cm central hemidiaphragm defect was noted. A composite bioabsorbable mesh (Phasix ST Mesh; BD, Franklin Lakes, $\mathrm{NJ}$ ) was tailored, appropriately oriented, and introduced into the abdomen. The defect was closed primarily using interrupted, figure-of-eight 2-0 nonabsorbable suture (ETHIBOND; Ethicon, Sommerville, NJ) buttressing the intraabdominal mesh and reinforced with intrathoracic mesh placement in a "sandwich" fashion (Video 1). A soft chest tube was placed to water-seal drainage, and $0.25 \%$ bupivacaine intercostal blocks were performed.
Estimated blood loss was $20 \mathrm{~mL}$. Duration of surgery was 181 minutes. The patient reported minimal pain in the immediate postoperative period. The chest tube was removed the morning of postoperative day 1 , and the patient was discharged the following morning. At 1 month and 6 months after surgery, no recurrence of hernia was observed (Figure 1).

\section{DISCUSSION}

The basic tenet of diaphragmatic hernia repair mandates a tension-free repair with or without reinforcement with prosthetic materials. ${ }^{4}$ Traditionally, central diaphragmatic hernias have been repaired transabdominally, owing to
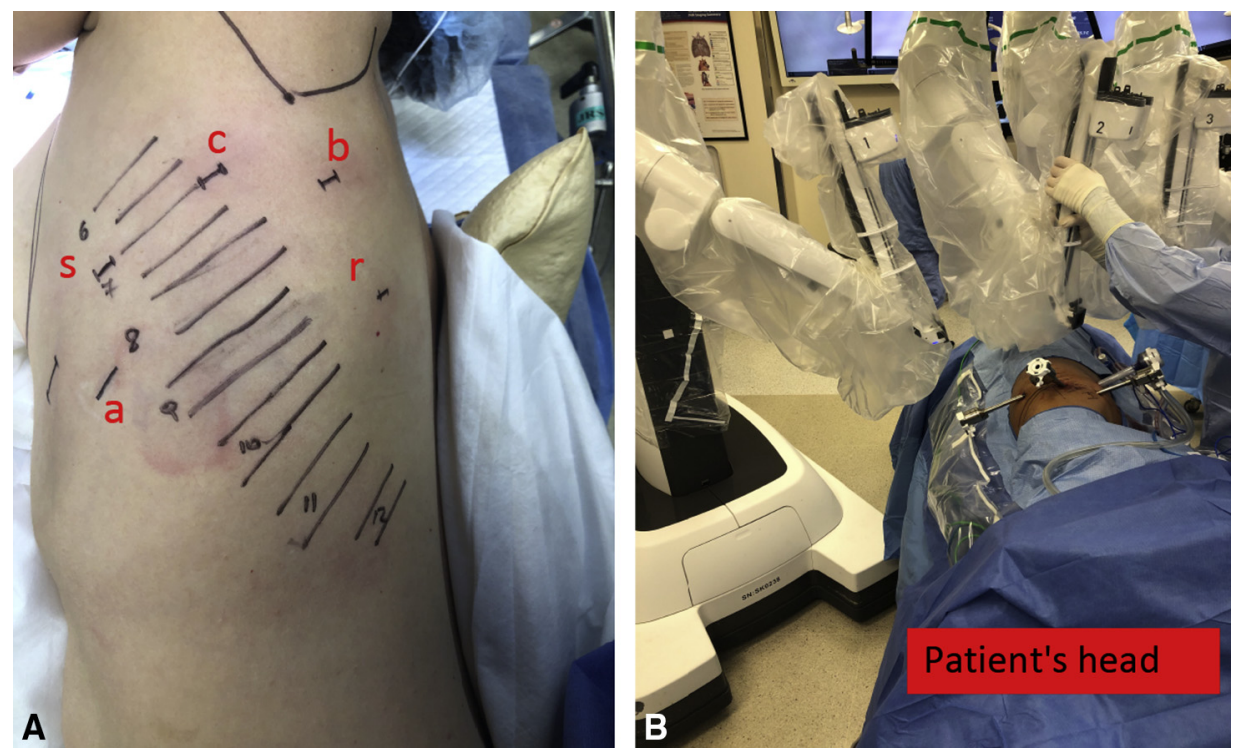

FIGURE 2. A, Port position ( $a$, assistant port; $b$, fenestrated bipolar; $c$, camera; $r$, retracting arm; $s$, monopolar spatula). B, Orientation of the robot; the legend indicates the position of the patient's head. 


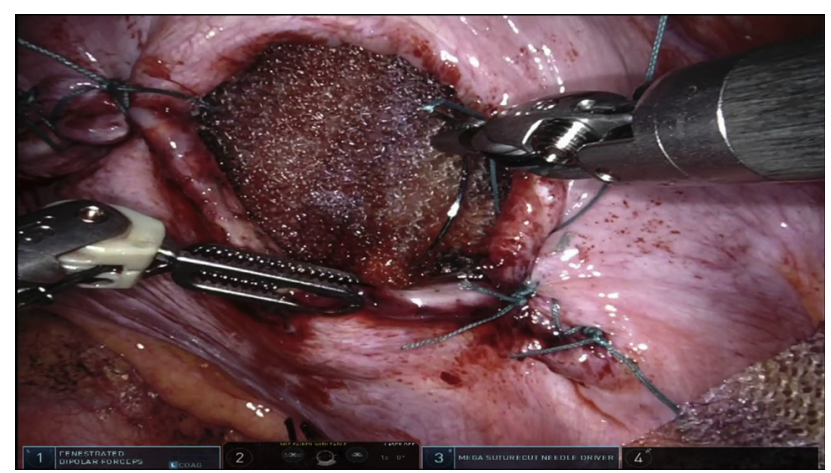

VIDEO 1. Robotic transthoracic diaphragmatic hernia repair. Video available at: https://www.jtcvs.org/article/S2666-2507(21)00585-X/fulltext.

surgeon familiarity, quick recovery, and the ability to place mesh intraabdominally to buttress the repair to withstand sudden increases of intraabdominal pressure. ${ }^{4,5}$ However, an extremely hostile abdomen conveys substantial risk of inadvertent injuries to the abdominal viscera. Debulking gynecologic surgery obliterates the peritoneal cavity and adds to the risk of intraoperative injury. Giant hernia with near-complete filling of the pleural cavity with abdominal viscera has also been traditionally regarded as a relative contraindication to transabdominal repair. Transthoracic repairs have mostly been performed via open thoracotomy, with few reports of robotic-assisted repairs. ${ }^{1-3}$

No randomized controlled trials exist exploring the benefit of mesh reinforcement of diaphragmatic hernia repairs. Unlike hiatal hernias, where the use of mesh has been shown to reduce short-term recurrences, ${ }^{6}$ there are no definitive data suggesting improved short-term or longterm protection from recurrence with the addition of mesh. If there is enough muscle redundancy, it is our preference to primarily close the defect to restore the normal anatomy of the diaphragm and to preserve the domain of the peritoneal and thoracic cavities. It is our preference to reinforce the repair with composite mesh, as the muscle is often weakened after stripping of the peritoneum and multiple abdominal surgeries. We prefer a composite mesh with an antiadhesion film coating to minimize adhesions to nearby viscera, ${ }^{7}$ which may make future intraabdominal or intrathoracic operations challenging. Although this was a left diaphragmatic hernia, conceivably the technique is applicable for right-sided repairs. The presence of the liver on the right side makes herniation of abdominal contents less likely. In the presence of liver herniation, meticulous attention to avoid bleeding while staying in the correct plane of dissection is paramount.

Proponents of robotic-assisted surgery cite superior visualization and improved ergonomics; drawbacks include perceived greater cost and longer duration of surgery. ${ }^{8}$ The case described here demonstrates that robotic-assisted repair is feasible and may be appropriate in select patients with diaphragmatic hernias, regardless of the size of the hernia. Furthermore, placement of intraabdominal mesh is feasible, and the repair can be reinforced with intrathoracic mesh. Robotic diaphragmatic hernia repair allows for minimal postoperative pain and hastened recovery.

\section{References}

1. Kim JK, Desai A, Kunac A, Merchant AM, Lovoulos C. Robotic transthoracic repair of a right-sided traumatic diaphragmatic rupture. Surg $J(N Y)$. 2020;6: e164-6.

2. Counts SJ, Saffarzadeh AG, Blasberg JD, Kim AW. Robotic transthoracic primary repair of a diaphragmatic hernia and reduction of an intrathoracic liver. Innovations (Phila). 2018;13:54-5.

3. Campbell S, Kipnis S. Robotic repair of an incarcerated right-sided diaphragmatic hernia after liver resection. Available at: https://www.ctsnet.org/article/roboticrepair-incarcerated-right-sided-diaphragmatic-hernia-after-liver-resection. Accessed June 9, 2021.

4. Blitz M, Louie BE. Chronic traumatic diaphragmatic hernia. Thorac Surg Clin. 2009;19:491-500.

5. Liao CH, Chu CH, Wu YT, Fu CY, Hsieh FJ, Wang SY, et al. The feasibility and efficacy of laparoscopic repair for chronic traumatic diaphragmatic herniation: introduction of a novel technique with literature review. Hernia. 2016;20:303.

6. Abdelmoaty WF, Dunst CM, Filicori F, Zihni AM, Davila-Bradley D, Reavis KM, et al. Combination of surgical technique and bioresorbable mesh reinforcement of the crural repair leads to low early hernia recurrence rates with laparoscopic paraesophageal hernia repair. J Gastrointest Surg. 2020;24:1477-81.

7. Deeken CR, Matthews BD. Characterization of the mechanical strength, resorption properties, and histologic characteristics of a fully absorbable material (poly-4-hydroxybutyrate-PHASIX mesh) in a porcine model of hernia repair. ISRN Surg. 2013;2013:238067.

8. Ricciardi S, Zirafa CC, Davini F, Melfi F. How to get the best from robotic thoracic surgery. J Thorac Dis. 2018;10(Suppl 8):S947-50. 